\title{
An assessment of hypercapnia-induced elevations in regional cerebral perfusion during combined orthostatic and heat stresses
}

\author{
Manabu Shibasaki ${ }^{*}$ (D), Kohei Sato², Ai Hirasawa ${ }^{3}$, Tomoko Sadamoto ${ }^{4}$, Craig G. Crandall ${ }^{5,6}$ and Shigehiko Ogoh
}

\begin{abstract}
We investigated that the effects of hypercapnia-induced elevations in cerebral perfusion during a heat stress on global cerebrovascular responses to an orthostatic challenge. Seven volunteers completed a progressive lower-body negative pressure (LBNP) challenge to presyncope during heat stress, with or without breathing a hypercapnic gas mixture. Administration of the hypercapnic gas mixture increased the partial pressure of end-tidal $\mathrm{CO}_{2}$ greater than pre-heat stress alone, and increased both internal carotid artery (ICA) and vertebral artery (VA) blood flows $(P<0.05)$. During LBNP, both ICA and VA blood flows with the hypercapnic gas mixture remained elevated relative to the control trial $(P<0.05)$. However, at the end of LBNP due to pre-syncopal symptoms, both ICA and VA blood flows decreased to similar levels between trials. These findings suggest that hypercapnia-induced cerebral vasodilation is insufficient to maintain cerebral perfusion at the end of LBNP due to pre-syncope in either the anterior or posterior vascular beds.
\end{abstract}

Keywords: Orthostatic tolerance, Presyncope, Hyperthermia, Cerebral blood flow, $\mathrm{CO}_{2}$ reactivity

\section{Introduction}

Passive heat stress reduces orthostatic tolerance [1, 2], but the physiological mechanism(s) responsible for this occurrence remains debatable. A primary mechanism leading to pre-syncopal symptoms is thought to cerebral hypoperfusion [3]. Head-up tilt or lower body negative pressure (LBNP) are often used to investigate physiological responses to orthostatic-induced fluid shifts in humans [4]. Hyperthermia associated with passive heat stress induces hyperventilation and associated hypocapnia-induced reductions in cerebral perfusion [5, 6]. Such hyperthermia-induced cerebral hypoperfusion may contribute to heat-induced reductions in orthostatic intolerance $[2,5]$. Since cerebral perfusion can be modulated through changes in arterial carbon dioxide tension $\left(\mathrm{PaCO}_{2}\right)[7]$, it may be that heat-induced orthostatic

*Correspondence: shiba@cc.nara-wu.ac.jp

${ }^{1}$ Department of Health Sciences, Faculty of Human Life and Environment, Nara Women's University, Kitauoya-Nishi Machi, Nara 630-8506, Japan

Full list of author information is available at the end of the article intolerance can be prevented by inhaling a hypercapnic gas mixture prior to the orthostatic challenge.

Indeed, Howden et al. demonstrated that the inhalation of a hypercapnic gas mixture improved orthostatic tolerance, simulated by LBNP, in normothermic subjects [8]. Given these findings, Lucas et al. addressed the same question but in heat-stressed subjects. In that study, cerebral perfusion was estimated by transcranial Dopplerderived middle cerebral artery blood velocity $\left(\mathrm{MCA}_{\mathrm{vel}}\right)$ [9]. Despite substantially elevated $\mathrm{MCA}_{\mathrm{vel}}$ secondary to inhalation of the hypercapnic gas mixture, LBNP tolerance was not improved in heat-stressed individuals relative to the trial without administering the hypercapnic gas mixture. However, a limitation of that protocol is the possibility that $\mathrm{MCA}_{\mathrm{vel}}$ was not representative of changes in global cerebral perfusion (i.e., $\mathrm{MCA}_{\mathrm{vel}}$ primary reflects blood flow to the anterior regions). Lewis et al. reported no difference between MCA and posterior cerebral artery (PCA) blood velocities during a normothermic orthostatic challenge [10]. In contrast, we observed different volumetric blood flow responses between the internal 
carotid artery (ICA) and the vertebral artery (VA) during progressive orthostatic stress, with VA blood flow being maintained while ICA blood flow decreased [11, 12]. Importantly, the regulation between ICA and VA blood flows during heat stress is different from that observed in normothermia [13].

Therefore, it is possible that cerebral perfusion in the posterior regions, supplied by the basilar and VA arteries, respond differently during combined heat and orthostatic stresses, relative to anterior regions (e.g., regions supplied by the MCA). Notably, such responses are speculative without volumetric assessments of regional blood flow during such stressors. We, therefore, hypothesized that the relative changes in volumetric cerebral blood flow between the anterior (ICA) and posterior cerebral arteries (VA) are different during combined heat and orthostatic stresses, and such differences are modified by a hypercapnic challenge (i.e., the inhalation of a hypercapnic gas mixture attenuates the decrease in cerebral blood flow in the anterior but not posterior regions).

\section{Methods}

\section{Ethical approval}

Experimental procedures and the protocol conformed to the Declaration of Helsinki and were approved by the Human Subjects Committee of Nara Women's University (14-01). Each subject provided written, informed consent after all potential risks and procedures were explained.

\section{Subjects}

Fourteen subjects enrolled in this study, but only ten completed both limbs of the protocol. Of those ten subjects, analyzable data were obtained from seven subjects due primarily to an inability to obtain images of sufficient quality at the termination of lower body negative pressure (LBNP) in three subjects. These seven subjects (four men and three women) had a mean \pm SD age of $21 \pm 1$ years, height of $163 \pm 9 \mathrm{~cm}$ and weight of $57 \pm 14 \mathrm{~kg}$. The subjects were free of any known cardiovascular or pulmonary disorders and were not using any prescribed or over-the-counter medications. In addition, subjects did not engaged in endurance training on a regular basis ( $<5 \mathrm{~h}$ /week). For female subjects, repeated testing was conducted at the same phase of the menstrual cycle, although menstrual cycle phase was not controlled. Subjects abstained from caffeinated beverages for $12 \mathrm{~h}$, and from strenuous physical activity and alcohol for at least $24 \mathrm{~h}$, before each trial.

\section{Experimental measurements}

At each experimental day, subjects emptied their bladder before being weighed to identify nude body mass.
Subjects, wearing short pants and underwear, were instrumented with ECG and skin temperature probes, and then dressed in a long-sleeved and long-legged, twopieced, tube-lined perfusion suit (Med-Eng, Ottawa, Canada) enabling the control of skin temperature and internal body temperature via changing the water temperature perfusing the suit. The suit covered the entire body, except for the head, face, hands, one arm, and feet. As index of internal body temperature, external canal temperature $\left(T_{\text {ca }}\right)$ was measured using an infrared temperature probe (CE Thermo, NIPRO Inc., Osaka, Japan). The validity of this measure was previously confirmed against esophageal temperatures [14, 15]. Whole-body mean skin temperature $\left(T_{\mathrm{sk}}\right)$ was measured from the electrical average of six thermocouples [16] fixed to the skin with porous tape. Heart rate (HR) was obtained from an electrocardiogram (Biomulti 1000, NEC, Tokyo, Japan) and intermittent arterial blood pressure was obtained by auscultation of the brachial artery via electrosphygmomanometry (STBP-780, Colin, Tokyo, Japan). Beat-to-beat arterial blood pressure from a finger cuff was measured and reconstructed to give brachial artery pressures (Portapress, FMS, Amsterdam, The Netherlands). Finger arterial pressure was used solely to aid in the detection of presyncope during LBNP, while measures from the brachial artery were used for data analysis. Thermal and hemodynamic data were acquired continuously at $50 \mathrm{~Hz}$ throughout the experiment (MP150, Biopac, Santa Barbara, CA, USA). The subject breathed through a mouthpiece attached to a low-resistance two-way valve and pressure flowmeter. The valve mechanism allowed the subjects to inspire either room air or a hypercapnic gas mixture $\left(5 \% \mathrm{CO}_{2}, 21 \%\right.$ oxygen, balanced nitrogen) from a 200-L Douglas bag. Gas samples were obtained at the mouthpiece from which $\mathrm{PetCO}_{2}$ was obtained. Respiratory variables throughout the experiment were recorded by an automatic breath-by-breath respiratory gas analyzing system (ARCO2000 MET; Arcosystem, Chiba, Japan).

\section{Doppler measurements}

The right ICA and left VA diameters and blood velocities were measured with two ultrasound systems (Vivid i; GE Healthcare, Tokyo, Japan), each equipped with a $10-\mathrm{MHz}$ linear transducer, from which volumetric blood flows were calculated. Right ICA measurements were performed $\sim 1.0-1.5 \mathrm{~cm}$ distal to the carotid bifurcation while left VA measures was obtained between the transverse processes of $\mathrm{C} 5$ and the subclavian artery. The systolic and diastolic diameters were measured from three cardiac cycles in each stage, from which the mean diameter (in centimeters) was calculated as: 
Mean diameter $=($ systolic diameter $\times 1 / 3)$

$$
+(\text { diastolic diameter } \times 2 / 3) .
$$

The time-averaged mean blood flow velocities were recorded and analyzed (in centimeters per second). Blood flow velocity measures were obtained from the average of $\sim 10-20$ cardiac cycles, to account for any effects caused by respiration. Subjects were excluded if we were unable to obtain velocity recordings for a minimum of 4 continuous cardiac cycles of sufficient quality that were analyzable throughout each perturbation. Great care was taken to ensure that the probe position was stable, the insonation angle did not vary (60 deg in most cases), and the sample volume was positioned in the center of the vessel and adjusted to cover the width of the vessel diameter. Finally, volumetric blood flow was calculated by multiplying the cross-sectional area $[\pi \times$ (mean diameter $/ 2)^{2}$ ] by mean blood flow velocity, as follows:

Blood flow $=$ mean blood flow velocity

$$
\times \text { area } \times 60(\mathrm{~mL} / \text { mintue }) \text {. }
$$

\section{Experimental protocol}

Subjects reported to the laboratory on two separate occasions. With each visit, subjects underwent a progressive LBNP trial while heat stressed either with or without inhalation of the hypercapnic gas mixture. The order of the experimental trials was randomized, and separated by a minimum of 3 days, with each trial being performed at the same time of day. After instrumentation, subjects were positioned in the LBNP box that was sealed at the level of the iliac crest. Subjects rested supine for at least $30 \mathrm{~min}$ prior to normothermic measurements (Norm baseline). Subjects were then passively heated by circulating warm water $\left(\sim 50{ }^{\circ} \mathrm{C}\right)$ through the suit until $T_{\text {ca }}$ increased by $\sim 1.2{ }^{\circ} \mathrm{C}$. Then, water temperature was slightly decreased to temper the rate of rise in $T_{\mathrm{ca}}$. When $T_{\text {ca }}$ was stable $\left(\sim 1.4{ }^{\circ} \mathrm{C}\right.$ above Norm baseline), baseline heat-stressed measurements (Heat baseline) were obtained prior to the onset of LBNP. Subsequently, beginning at $20 \mathrm{mmHg}, 3$-min stages of LBNP were applied at $10 \mathrm{mmHg}$ per stage until the occurrence of syncopal symptoms. In the $\mathrm{CO}_{2}$ trial, subjects inhaled the hypercapnic gas mixture 2 min before the onset of the LBNP challenge, with $1 \mathrm{~min}$ of hypercapnic data $\left(\mathrm{Heat} \mathrm{CO}_{2}\right)$ collected before the onset of LBNP. The LBNP challenge was then applied with the subject continuing to breathe the hypercapnic gas mixture or room air throughout. Test termination was based on the following criteria: continued self-reporting by the subject of feeling faint or feeling like he/she could no longer tolerate LBNP, pallor, diaphoresis, rapid and progressive decrease in blood pressure resulting in systolic blood pressure being less than $70 \mathrm{mmHg}$, and/or relative bradycardia accompanied with narrowing of pulse pressure. Typically, a combination of the aforementioned conditions was observed at the cessation of the tolerance test, with a reduction in pulse pressure along with relative bradycardia being the most common observations. The total time of each LBNP challenge was measured and used to determine a cumulative stress index (CSI) by summing the product of the negative pressure and duration at each stage of LBNP, in minutes and fraction of minutes (e.g., $20 \mathrm{mmHg} \times 3 \mathrm{~min}$ $+30 \mathrm{mmHg} \times 3 \mathrm{~min}+40 \mathrm{mmHg} \times 3 \mathrm{~min}$, etc.) until the test was terminated [17].

\section{Data analysis}

In both trials, $60 \mathrm{~s}$ of data were averaged for Norm baseline before the start of whole-body heating. Heat baseline values were averaged from $60 \mathrm{~s}$ of data after $T_{\mathrm{ca}}$ had increased by $\sim 1.4{ }^{\circ} \mathrm{C}$. Thermal (i.e., $T_{\mathrm{sk}}$ and $T_{\mathrm{ca}}$ ), hemodynamic (heart rate; HR and mean arterial pressure; MAP), and respiratory (minute ventilation; $\mathrm{VE}$, end-tidal carbon dioxide partial pressure $\mathrm{P}_{\mathrm{ET}} \mathrm{CO}_{2}$, and respiratory rate; $\mathrm{RR}$ ) variables before application of the hypercapnic gas mixture were evaluated by a two-way repeated measures analysis of variance (ANOVA) with main factors of time (Norm baseline vs. Heat baseline) and treatment days. In the $\mathrm{CO}_{2}$ trial, the hypercapnic data $\left(\mathrm{Heat} \mathrm{CO}_{2}\right)$ were evaluated by paired T test (Heat baseline vs. Heat $\mathrm{CO}_{2}$ ) to confirm the effect of the hypercapnic gas on the assessed variables. For the analyses of the responses to the LBNP challenge, Pre-LBNP data (i.e., prior to the LBNP challenge) were used from Heat baseline for the Air trial while Heat $\mathrm{CO}_{2}$ baseline data were used for the $\mathrm{CO}_{2}$ trial. The LBNP analysis included averaged responses during the last $12 \mathrm{~s}$ before the occurrence of any pre-syncopal symptoms (primarily the point just before a reduction in heart rate during the LBNP challenge; termed severe LBNP). Tolerance data were obtained by averaging the responses during the last $12 \mathrm{~s}$ before the end of the LBNP challenge due to syncopal symptoms. A three-way repeated measures ANOVA with main factors of region (ICA vs. VA), time (pre-LBNP, severe LBNP, end of LBNP) and experimental day (Air vs. $\mathrm{CO}_{2}$ ) was used only to compare relative changes in ICA and VA blood flows during the LBNP challenge. Two-way repeated measures ANOVA with main factors of time (pre-LBNP, severe LBNP, end of LBNP) and experimental day (Air vs. $\mathrm{CO}_{2}$ ) were used to identify differences in the assessed variables between the Air and $\mathrm{CO}_{2}$ trials. Any main effect differences were further explored via Student-Newman-Keuls post hoc tests. ICA and VA blood flow were normalized relative to the respective normothermic baselines, thereby enabling a comparison of regional differences. Paired $t$ tests 
were used to identify differences in CSI between Air and the $\mathrm{CO}_{2}$ trials. The $\alpha$ level for all analyses was set at 0.05 . Results are reported as mean \pm SD.

\section{Results}

\section{Physiological responses prior to the LBNP challenge}

Pre-heat stress thermal, hemodynamic, and respiratory baseline measures were not difference between the trials (Table 1). Heat stress increased $T_{\mathrm{sk}}$ similarly between trials (Table 1) and increased $T_{\mathrm{ca}}$ by $1.40 \pm 0.07{ }^{\circ} \mathrm{C}$ (Air trial) and $1.41 \pm 0.14{ }^{\circ} \mathrm{C}\left(\mathrm{CO}_{2}\right.$ trial $)$. Absolute and relative reductions in body mass as a result of the heat stress were similar between trials (Air trial: $56.7 \pm 14.1$ to $55.9 \pm 13.9 \mathrm{~kg} ; \mathrm{CO}_{2}$ trial: $56.9 \pm 14.3$ to $56.0 \pm 14.1 \mathrm{~kg}$ ). Changes in hemodynamic and respiratory variables as a result of the heat stress were similar between these trials prior to inhaling the hypercapnic gas mixture (Table 1). During heating, absolute VA blood flow was slightly higher in the Air trial relative to the $\mathrm{CO}_{2}$ trial, but normalized responses (i.e., \%baseline data) were similar between trials. Heat stress decreased ICA, but not VA, blood flows. Inhaling the hypercapnic gas mixture increased ICA and VA blood flows by 1.3-fold relative to the respective normothermic baseline values, increased respiratory responses, while temperatures, HR and MAP were maintained (Table 1).

\section{Responses to the LBNP challenge}

LBNP tolerance was similar between the trials (CSI for Air: $178.5 \pm 114.5 \mathrm{mmHg} \times \min$ vs. CSI for $\mathrm{CO}_{2}$ : $269.5 \pm 171.6 \mathrm{mmHg} \times \min , P=0.272)$. Consistent with that observation, the final LBNP stage achieved was also not different between trials (Air: $34 \pm 10$ vs. $\mathrm{CO}_{2}$ : $44 \pm 11 \mathrm{mmHg}, P=0.11)$. $T_{\mathrm{ca}}$ was maintained throughout LBNP in both the Air and $\mathrm{CO}_{2}$ trials (end of LBNP: $38.6 \pm 0.3{ }^{\circ} \mathrm{C}$ vs. $38.6 \pm 0.2{ }^{\circ} \mathrm{C}$, respectively), while $T_{\mathrm{sk}}$ was slightly decreased for the $\mathrm{CO}_{2}$ trials (end of LBNP: $38.8 \pm 0.8{ }^{\circ} \mathrm{C}$ vs. $38.6 \pm 1.5{ }^{\circ} \mathrm{C}$, respectively). The LBNP

Table 1 Thermal, hemodynamic, respiratory variables, and blood flows

\begin{tabular}{|c|c|c|c|c|c|c|c|c|}
\hline & \multirow[t]{2}{*}{ Condition } & \multicolumn{3}{|l|}{ Stage } & \multicolumn{3}{|l|}{ ANOVA } & \multirow[t]{2}{*}{$T$ test } \\
\hline & & Norm baseline & Heat baseline & Heat- $\mathrm{CO}_{2}$ & Condition & Stage & Interaction & \\
\hline \multirow[t]{2}{*}{$T_{\text {sk }{ }^{\prime}}{ }^{\circ} \mathrm{C}$} & Air & $33.8 \pm 0.7$ & $39.0 \pm 0.7$ & $38.9 \pm 1.0$ & 0.959 & $<0.001$ & 0.471 & 0.724 \\
\hline & $\mathrm{CO}_{2}$ & $33.9 \pm 0.5$ & $38.9 \pm 1.0$ & & & & & \\
\hline \multirow[t]{2}{*}{$T_{\mathrm{Ca},}{ }^{\circ} \mathrm{C}$} & Air & $37.2 \pm 0.3$ & $38.6 \pm 0.2$ & $38.7 \pm 0.1$ & 0.775 & $<0.001$ & 0.365 & 0.060 \\
\hline & $\mathrm{CO}_{2}$ & $37.3 \pm 0.1$ & $38.6 \pm 0.1$ & & & & & \\
\hline \multirow[t]{2}{*}{$\mathrm{HR}, \mathrm{bpm}$} & Air & $61.7 \pm 7.1$ & $104.3 \pm 11.9$ & $102.6 \pm 8.7$ & 0.083 & $<0.001$ & 0.128 & 0.067 \\
\hline & $\mathrm{CO}_{2}$ & $60.4 \pm 7.5$ & $98.3 \pm 7.4$ & & & & & \\
\hline \multirow[t]{2}{*}{ MAP, mmHg } & Air & $76.4 \pm 8.5$ & $77.9 \pm 8.9$ & $86.3 \pm 8.7$ & 0.207 & 0.717 & 0.171 & 0.045 \\
\hline & $\mathrm{CO}_{2}$ & $85.1 \pm 14.4$ & $82.0 \pm 9.1$ & & & & & \\
\hline \multirow[t]{2}{*}{$V E, L / m i n$} & Air & $7.1 \pm 1.5$ & $9.3 \pm 2.8$ & $17.0 \pm 4.4$ & 0.876 & 0.011 & 0.971 & 0.002 \\
\hline & $\mathrm{CO}_{2}$ & $7.3 \pm 1.9$ & $9.6 \pm 2.0$ & & & & & \\
\hline \multirow[t]{2}{*}{$\mathrm{RR}, n$} & Air & $14.7 \pm 2.4$ & $15.9 \pm 5.0$ & $18.1 \pm 5.9$ & 0.227 & 0.309 & 0.894 & 0.414 \\
\hline & $\mathrm{CO}_{2}$ & $14.1 \pm 2.6$ & $16.4 \pm 3.4$ & & & & & \\
\hline \multirow[t]{2}{*}{$\mathrm{P}_{\mathrm{ET}} \mathrm{CO}_{2}, \mathrm{mmHg}$} & Air & $42.9 \pm 2.6$ & $40.0 \pm 5.4$ & $49.3 \pm 3.6$ & 0.876 & 0.054 & 0.902 & 0.002 \\
\hline & $\mathrm{CO}_{2}$ & $43.0 \pm 2.4$ & $40.3 \pm 4.0$ & & & & & \\
\hline \multirow[t]{2}{*}{$V A, m L / m i n$} & Air & $143.5 \pm 43.8$ & $141.8 \pm 51.5$ & $171.1 \pm 51.7$ & 0.045 & 0.439 & 0.619 & 0.010 \\
\hline & $\mathrm{CO}_{2}$ & $132.0 \pm 41.2$ & $123.0 \pm 34.4$ & & & & & \\
\hline \multirow[t]{2}{*}{ ICA, $\mathrm{mL} / \mathrm{min}$} & Air & $398.2 \pm 72.9$ & $352.2 \pm 87.8$ & $459.8 \pm 100.5$ & 0.586 & 0.039 & 0.645 & 0.019 \\
\hline & $\mathrm{CO}_{2}$ & $369.8 \pm 70.8$ & $337.9 \pm 73.0$ & & & & & \\
\hline \multirow[t]{2}{*}{ \%VA, \%baseline } & Air & 100 & $99.2 \pm 18.7$ & $133.0 \pm 33.1$ & 0.632 & 0.582 & 0.632 & 0.010 \\
\hline & $\mathrm{CO}_{2}$ & 100 & $94.7 \pm 17.7$ & & & & & \\
\hline \multirow[t]{2}{*}{$\% \mid C A, \%$ baseline } & Air & 100 & $88.7 \pm 16.2$ & $127.0 \pm 35.7$ & 0.685 & 0.040 & 0.685 & 0.040 \\
\hline & $\mathrm{CO}_{2}$ & 100 & $91.5 \pm 9.5$ & & & & & \\
\hline
\end{tabular}

Blood flow values are depicted in absolute units and relative changes from the normothermic baseline prior to LBNP challenge. Values are mean \pm SD were averaged from steady-state supine data at normothermic (Norm baseline) and during heat stress (Heat baseline). Prior to the LBNP challenge, the hypercapnic gas was applied in the $\mathrm{CO}_{2}$ trial $\left(\mathrm{Heat} \mathrm{CO}_{2}\right)$ only. $T_{\text {ske }}$ mean skin temperature; $T_{c,}$ external canal temperature; $\mathrm{VE}$, minute ventilation; $\mathrm{RR}$, respiratory rate; $\mathrm{P}_{\mathrm{ET}} \mathrm{CO}_{2}$, partial pressure of end-tidal carbon dioxide; MAP, mean arterial blood pressure; HR, heart rate; ICA, internal carotid artery; VA, vertebral artery blood flow. ICA and VA blood flows were depicted in absolute units and relative changes (i.e., normalized relative to the respective normothermic baselines). Subjects performed two progressive lower body pressure during heat stress with ( $\mathrm{CO}_{2}$ trial) or without (Air trial) an application of hypercapnic gas mixture on different day. Thermal, hemodynamic, respiratory, and cerebrovascular variables were evaluated by a two-way repeated measures analysis of variance (ANOVA). The paired $T$ test (Heat baseline vs. Heat $\mathrm{CO}_{2}$ ) performed to evaluate the effect of inhaling hypercapnic gas on these variables 
challenge increased HR, but MAP was maintained in both trials prior to the onset of pre-syncope symptoms (Fig. 1). At the end of LBNP, MAP was significantly decreased in both trials, with the magnitude of this reduction being similar between trials. $\mathrm{P}_{\mathrm{ET}} \mathrm{CO}_{2}$ in the Air trial decreased to $31.7 \pm 6.6 \mathrm{mmHg}$ at the end of LBNP. For the $\mathrm{CO}_{2}$ trial, administration of the hypercapnic gas

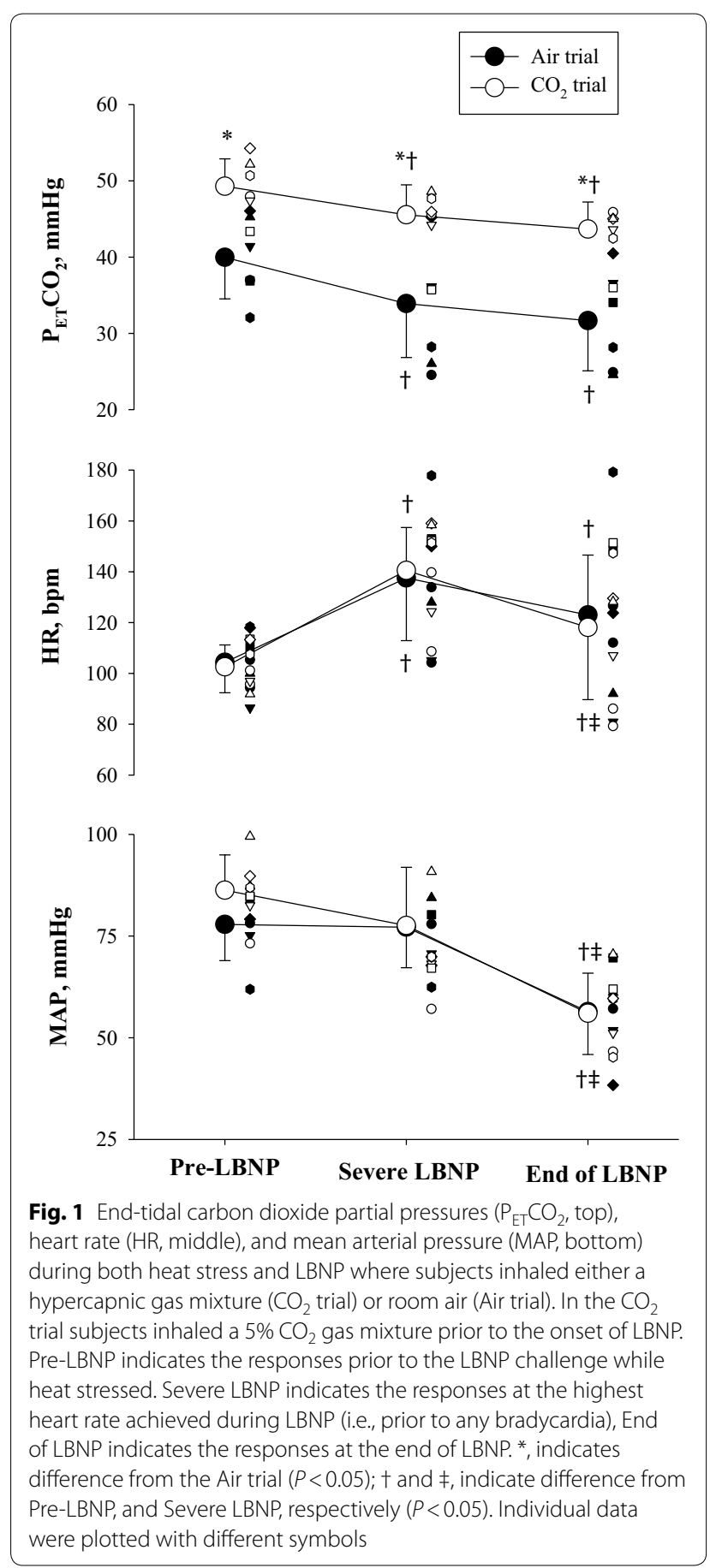

mixture increase $\mathrm{P}_{\mathrm{ET}} \mathrm{CO}_{2}$ to above normothermic baseline, with $\mathrm{P}_{\mathrm{ET}} \mathrm{CO}_{2}$ at the end of LBNP $(43.7 \pm 3.6 \mathrm{mmHg})$ being similar to $\mathrm{P}_{\mathrm{ET}} \mathrm{CO}_{2}$ at normothermic baseline $(43.0 \pm 2.4 \mathrm{mmHg})$.

ANOVAs for relative changes in blood flows during LBNP, with or without inhaling the hypercapnic gas mixture, showed a significant main effect of time and experimental day, but not for regional cerebral perfusion (see Figs. 2, 3 and Table 2). Figure 2 shows the relative changes in ICA and VA blood flows to the LBNP challenges. Before LBNP, both ICA and VA blood flows in the $\mathrm{CO}_{2}$ trial were elevated above the normothermic

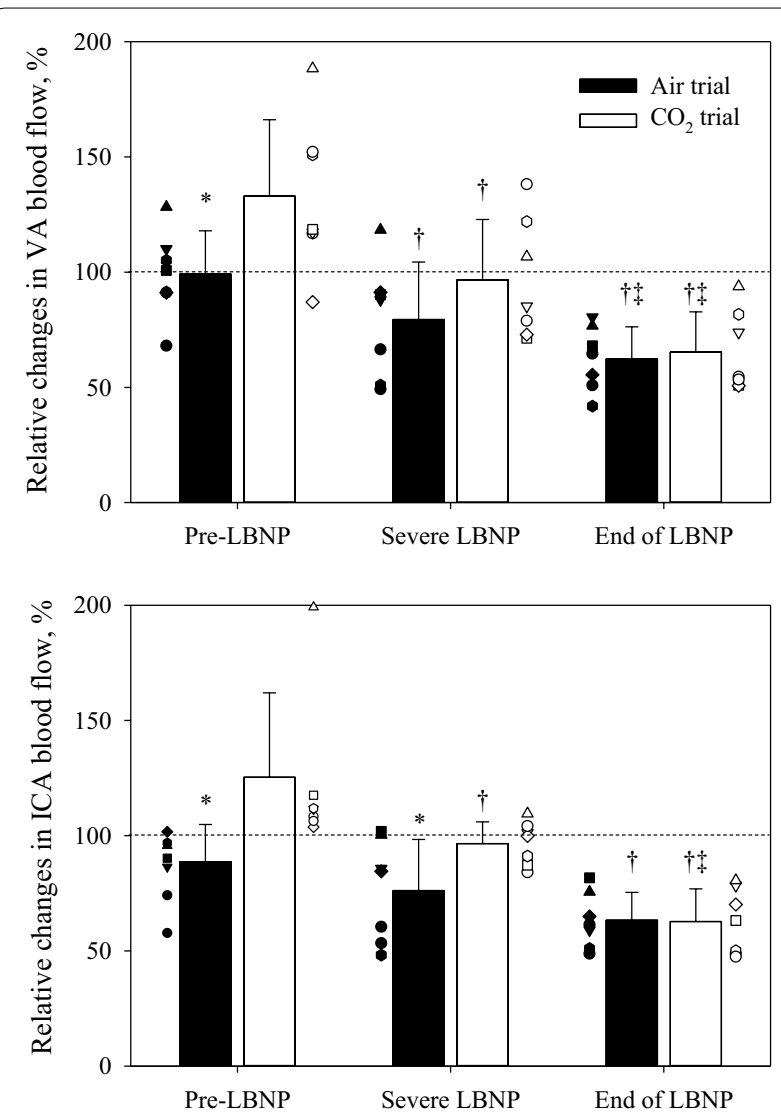

Fig. 2 Relative changes in vertebral artery (VA, top) and internal carotid artery (ICA, bottom) blood flow at each LBNP stage (Pre-LBNP, Severe LBNP and End of LBNP), with (open) or without (solid) the hypercapnic gas. Application of the hypercapnic gas mixture increased both ICA and VA blood flows. LBNP decreased blood flow to both regions (i.e., Severe LBNP). However, at Severe LBNP in the hypercapnic trial, both ICA and VA blood flows were at a similar level relative to normothermic baseline (indicated by the dashed horizontal line), whereas in the Air trial those were values were below normothermic baseline. At the end of LBNP, due to presyncope, both ICA and VA blood flows were equivalent and both were lower than normothermic baseline. ${ }^{*} P<0.05$ different from the Air trial; ${ }^{\dagger} P<0.05$ different from Pre-LBNP: ${ }^{\ddagger} P<0.05$ different from Severe LBNP 


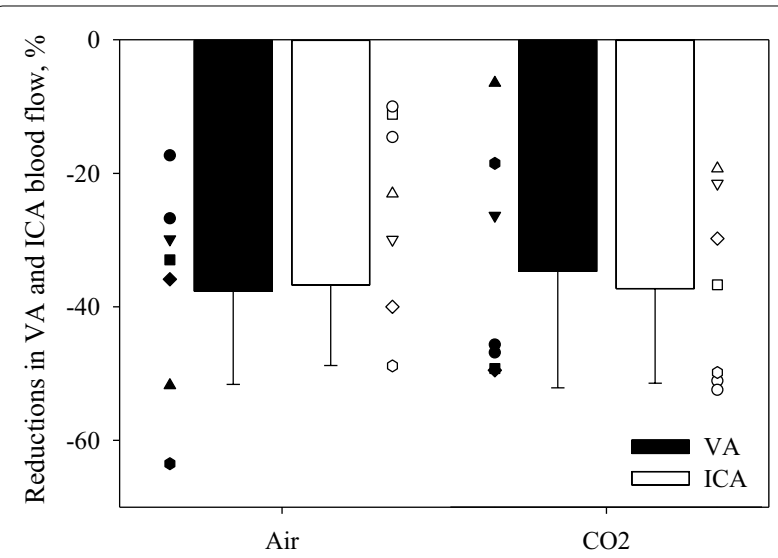

Fig. 3 Reductions in relative changes of vertebral artery (VA, top) and internal carotid artery (ICA, bottom) blood flow to LBNP stress with (open) or without (solid) the hypercapnic gas. Both ICA and VA blood flows were decreased below normothermic baseline at the end of LBNP

baseline and were both greater relative to the same time point in the Air trial. LBNP decreased both blood flows in each trial, and at the end of LBNP both ICA and VA blood flows had decreased below the respective normothermic baselines (dashed line in Fig. 2); this was accomplished by both diameters and velocities of ICA and VA decreasing at the end of LBNP relative to pre-LBNP during both Air and $\mathrm{CO}_{2}$ trials (Table 2). The relative reduction in ICA and VA blood flows to LBNP from the normothermic baseline were not different between trials, with ICA and VA blood flows decreasing to $63.3 \pm 12.1 \%$ and $62.4 \pm 14.0 \%$ in the Air trial and to $62.7 \pm 14.2 \%$ and
$65.3 \pm 17.5 \%$ in the $\mathrm{CO}_{2}$ trial, respectively. ANOVAs for the reductions in relative blood flows due to LBNP, with or without inhaling the hypercapnic gas mixture, showed no significant main effect of regions $(P=0.843)$, experimental day $(P=0.811)$, or interaction $(P=0.664)$. Thus, regardless of the inhaled gas, no regional differences between ICA and VA blood flows, normalized by normothermic baseline values, were observed (Fig. 3).

\section{Discussion}

Global cerebral blood flow responses during an LBNP tolerance test in heat-stressed subjects were evaluated with and without the administration of a hypercapnic gas mixture. The main observations of this study are: (1) despite an increase in both VA and ICA blood flows via an inhaling a hypercapnic gas mixture while heat stressed, LBNP tolerance was not improved; (2) there was no regional difference in the magnitude of the relative reduction in cerebral perfusion between ICA and VA vessels during LBNP for either the Air and $\mathrm{CO}_{2}$ trials; (3) at the end of LBNP, both ICA and VA blood flows decreased below the normothermic baseline values regardless of the inhaled gas. These findings show that the application of a hypercapnic gas mixture increased both anterior and posterior cerebral blood flow during heat stress, but this stimulus was insufficient to maintain cerebral perfusion at presyncope.

Excessive increases of core/internal temperature due to passive heat stress greatly reduce orthostatic tolerance $[1,2]$. Passive heating decreases total peripheral vascular resistance, and also promotes hyperventilation-induced hypocapnia, particularly when core/internal temperature

Table 2 Internal carotid artery (ICA) and vertebral artery (VA) responses during lower body negative pressure (LBNP)

\begin{tabular}{|c|c|c|c|c|c|c|c|}
\hline & \multirow[t]{2}{*}{ Condition } & \multicolumn{3}{|l|}{ Stage } & \multicolumn{3}{|l|}{ ANOVA } \\
\hline & & Pre-LBNP & Severe LBNP & End of LBNP & Condition & Stage & Interaction \\
\hline \multirow[t]{2}{*}{ VA blood flow, $\mathrm{mL} / \mathrm{min}$} & Air & $141.8 \pm 51.5$ & $116.8 \pm 53.5^{\dagger}$ & $92.2 \pm 42.0^{\dagger \neq}$ & 0.465 & $<0.001$ & 0.102 \\
\hline & $\mathrm{CO}_{2}$ & $171.1 \pm 51.7$ & $124.8 \pm 42.3^{\dagger}$ & $85.5 \pm 34.8^{\dagger \neq}$ & & & \\
\hline \multirow[t]{2}{*}{ ICA blood flow, $\mathrm{mL} / \mathrm{min}$} & Air & $352.2 \pm 87.8$ & $302.5 \pm 104.5$ & $251.2 \pm 66.2^{\dagger}$ & 0.153 & $<0.001$ & 0.061 \\
\hline & $\mathrm{CO}_{2}$ & $459.8 \pm 100.5^{*}$ & $356.9 \pm 76.7^{\dagger}$ & $229.6 \pm 54.1^{t \neq}$ & & & \\
\hline \multirow[t]{2}{*}{ VA diameter, cm } & Air & $0.37 \pm 0.05$ & $0.36 \pm 0.04$ & $0.35 \pm 0.05^{\dagger}$ & 0.519 & $<0.001$ & 0.861 \\
\hline & $\mathrm{CO}_{2}$ & $0.36 \pm 0.05$ & $0.35 \pm 0.04^{\dagger}$ & $0.34 \pm 0.05^{\dagger}$ & & & \\
\hline \multirow[t]{2}{*}{ ICA diameter, cm } & Air & $0.49 \pm 0.05$ & $0.47 \pm 0.05$ & $0.46 \pm 0.04^{\dagger}$ & 0.901 & 0.001 & 0.762 \\
\hline & $\mathrm{CO}_{2}$ & $0.49 \pm 0.04$ & $0.48 \pm 0.05$ & $0.46 \pm 0.06^{\dagger}$ & & & \\
\hline \multirow[t]{2}{*}{ VA velocity, $\mathrm{cm} / \mathrm{s}$} & Air & $21.86 \pm 3.06$ & $18.45 \pm 5.58^{\dagger}$ & $15.50 \pm 3.95^{\dagger}$ & 0.297 & $<0.001$ & 0.063 \\
\hline & $\mathrm{CO}_{2}$ & $27.43 \pm 6.24$ & $21.75 \pm 6.67^{\dagger}$ & $15.39 \pm 2.81^{\dagger \neq}$ & & & \\
\hline \multirow[t]{2}{*}{ ICA velocity, $\mathrm{cm} / \mathrm{s}$} & Air & $31.66 \pm 6.13$ & $28.98 \pm 8.11$ & $24.61 \pm 5.06^{\dagger}$ & 0.206 & $<0.001$ & 0.043 \\
\hline & $\mathrm{CO}_{2}$ & $40.75 \pm 6.91^{*}$ & $34.02 \pm 10.6^{\dagger}$ & $22.77 \pm 4.52^{\dagger \neq}$ & & & \\
\hline
\end{tabular}

Values are mean $\pm S D$ that were averaged from steady-state supine data pre-lower body negative pressure application (Pre-LBNP), just before the occurrence of any pre-syncopal symptoms (Severe LBNP), and at the end of the LBNP challenge due to syncopal symptoms (End of LBNP). ICA: internal carotid artery, VA: vertebral artery (VA). ${ }^{*} P<0.05$, difference between Air and $\mathrm{CO}_{2} ;{ }^{\dagger} P<0.05$, difference from Pre-LBNP; ${ }^{\ddagger} P<0.05$, difference from Severe LBNP 
increase over $\sim 1.0{ }^{\circ} \mathrm{C}$, resulting in cerebral hypoperfusion [18-20]. Since cerebral hypoperfusion leads to presyncope symptoms [3], it was previously hypothesized that maintaining cerebral perfusion via inhaling hypercapnic gas in heat-stressed individuals would improve orthostatic intolerance [9]. However, Lucas et al. did not observe such a response, as elevating $\mathrm{MCA}_{\mathrm{vel}}$ via inhaling hypercapnic gas did not alter LBNP tolerance in heatstressed individuals [9]. They concluded that preserving cerebral perfusion does not influence orthostatic tolerance during hyperthermic condition. Consistent with the observations of Lucas et al., we also did not see differences in LBNP tolerance between trials (i.e., with or without the inhaling hypercapnic gas), despite $\mathrm{P}_{\mathrm{ET}} \mathrm{CO}_{2}$ being maintained at or above pre-heat level throughout LBNP in the $\mathrm{CO}_{2}$ trial [9]. However, the unique aspect of the present study is the assessment of regional cerebrovascular beds both to the hypercapnic challenge while heat stressed, and throughout subsequent LBNP to tolerance.

Blood supply to the brain originates not only from the ICA, but also the VA, with $\sim 65-70 \%$ of global cerebral blood flow being supplied by the ICA and the residual $\sim 25-30 \%$ being supplied by the VA [21]. The ICA provides blood to the anterior cerebral circulation through the MCA and anterior cerebral artery, while the VA merges with the basilar artery to provide blood to the posterior cerebral circulation, including the medulla oblongata, hypothalamus, thalamus, brainstem and cerebellum [22]; regions that are recognized to control autonomic function. In normothermia, we previously observed that orthostatic stress decreases ICA blood flow, while VA blood flow is generally maintained in tolerant subjects (i.e., no observation of pre-syncope symptoms) [11, 23]. Although speculative, given these previous observations, coupled with no regional difference in cerebral perfusion in pre-syncope subjects during heat stress, further investigation is needed to clarify the rationale of regional cerebral perfusion in orthostatic tolerance.

Previously, we also observed that cerebrovascular $\mathrm{CO}_{2}$ reactivity in the VA was smaller than that in the ICA, regardless of thermal status [13]. Given those observations, we expected differential changes in VA and ICA blood flows to the imposed challenges in the present study, culminating in VA blood flow in the $\mathrm{CO}_{2}$ trial not being maintained (or maintained less) relative to ICA blood flow during combined heat and LNBP stress. Contrary to that hypothesis, both VA and ICA blood flows decreased during the LBNP challenge, and culminated in a similar relative reduction in ICA and VA blood flows at the end of LBNP, regardless of the administration of the hypercapnic gas mixture. Nevertheless, these results suggest that volumetric changes in global cerebral perfusion are important when assessing orthostatic tolerance in the heat-stressed individuals.

A previous study reported that $\mathrm{MCA}_{\mathrm{vel}}$ remained greater than normothermic baseline at the end of LBNP during a hypercapnic challenge of heat-stressed individuals [9], which is inconsistent with our results. Recent findings raise a question about the reliability of transcranial Doppler-derived indices of CBF because the diameter of MCA can change with substantial changes in $\mathrm{PaCO}_{2}[24$, 25]. Also, van Helmond indicated that $\mathrm{MCA}_{\mathrm{vel}}$ during combined stresses, such as LBNP under hypoxia, might underestimate $\mathrm{CBF}$ because these stresses might change MCA diameter [26]. Therefore, the present protocol evaluated cerebral perfusion via volumetric changes in flow from regions perfused by both the ICA and VA, culminating in the observation that blood flows to both regions decreased below the respective normothermic baselines at the end of LBNP regardless of inhaled gas. Of note, we observed that the diameter of both the ICA and VA decreased during LBNP in both trials, while the velocities also decreased. Thus, under the imposed combined stresses, hypercapnia-induced increases in cerebral blood flow, due to cerebral vasodilation, is apparently overridden by reductions in perfusion pressure and/or accompanying cerebral vasoconstriction resulting in global decreases in cerebral blood flow. These findings suggest that regional cerebral perfusion cannot be maintained during combined heat and orthostatic stresses despite cerebral blood flow being elevated via the inhalation of a hypercapnic gas mixture.

There are two possible mechanisms for the observed heat stress-induced cerebral hypoperfusion, that is hypocapnia and hypotension $[6,10]$. Either breathing a hypercapnic gas mixture or expansion of blood volume can attenuate cerebral hypoperfusion during a hypotensive episode. Based upon the present results and Lucas et al's prior observation [9], an elevated cerebral perfusion due to breathing a hypercapnic gas mixture is unlikely to improve orthostatic tolerance in hyperthermic individuals. In contrast, acute volume expansion improves orthostatic tolerance in hyperthermic subjects [27], while a follow-up study demonstrated that such acute volume expansion attenuates the reduction in cerebral perfusion during combined heat and orthostatic stresses [28]. These observations suggest that in a case of acute dehydration, acute volume expansion is capable of improving orthostatic tolerance perhaps by preserving cerebral perfusion. However, a recent study investigated the interactive effects of incremental heat stress, orthostatic stress, and hypohydration, and suggested that hypohydration before heat stress does not influence the cerebrovascular or cardiovascular responses to LBNP [29]. Therefore, the mechanism(s) 
of orthostatic intolerance in the heat-stressed human remains unknown.

\section{Limitation of this study}

A previous study reported that $\mathrm{MCA}_{\mathrm{vel}}$ remained greater at the end of LBNP due to syncopal symptoms [9], whereas ICA blood flow in this study was decreased below the normothermic baseline. Despite administration of the hypercapnic gas, $\mathrm{P}_{\mathrm{ET}} \mathrm{CO}_{2}$ decreased slightly during LBNP, but this value did not decrease below $\mathrm{P}_{\mathrm{ET}} \mathrm{CO}_{2}$ at normothermic baseline. Though such reductions in $\mathrm{P}_{\mathrm{ET}} \mathrm{CO}_{2}$ might decrease ICA blood flow, $\mathrm{P}_{\mathrm{ET}} \mathrm{CO}_{2}$ at the end of LBNP was significantly greater in the $\mathrm{CO}_{2}$ trial relative to the Air trial. Nevertheless, absolute ICA blood flow in the $\mathrm{CO}_{2}$ trial was similar with that in the Air trial at the end of LBNP, indicating that regardless of inhaled hypercapnic gas mixture ICA blood flow is not preserved during a profound orthostatic stress. Blood flow evaluation by duplex ultrasonography has the advantage of allowing simultaneous quantification of the vessel diameter and the blood velocity (i.e., volumetric assessment), which cannot be obtained by using the transcranial Doppler (TCD) measurement technique. However, given that continuous volumetric flow measures is challenging, particularly at low perfusion states such as LBNP, we could not ascertain the dynamics of ICA and VA blood flow responses precisely at the onset of presyncope. Also, VA and ICA ultrasound measures were performed on the left and right side, respectively, recognizing that the left VA blood flow and diameter are slightly greater relative to the right VA blood flow and diameter [12]. This approach was selected to prioritize obtaining clear blood flow waveforms over assessing vessels ipsilaterally. Another approach to evaluate the control of the cerebral vasculature is to perform transfer function analyses (TFA) between changes in cerebral perfusion and arterial blood pressure. However, TFA was not possible in the present trial due to the short time period of each LBNP state [30]. Finally, 14 volunteers participated in this study, with 10 subjects completing both LBNP trials, and just 7 providing analyzable data. Such a small sample size might result in beta errors in some results that were found to not be statistically significant.

\section{Conclusion}

Global cerebral perfusion during LBNP in heat-stressed subjects was evaluated by volumetric changes of ICA and VA blood flows using duplex ultrasonography. An administration of hypercapnic gas increased blood flows through both vessels. Regardless of the inhaled hypercapnic gas mixture, ICA and VA blood flows decreased below their respective normothermic baselines at the end of LBNP, when pre-syncopal symptoms were evident. Thus the present results confirm that breathing hypercapnic gas mixture will not prevent orthostatic intolerance in the hyperthermic individuals. Moreover, regional difference in cerebral perfusion was not observed at the end of LBNP, regardless of whether $\mathrm{PaCO}_{2}$ is elevated, and global indices of cerebral perfusion were not maintained during severe orthostatic stress when combined with a heat stress.

\section{Abbreviations \\ LBNP: Lower-body negative pressure; ICA: Internal carotid artery; VA: Vertebral artery; MCA: Middle cerebral artery; PCA: Posterior cerebral artery; MCA $_{\text {vel: }}$ : Middle cerebral artery blood velocity; $T_{\text {ca: }}$ : External canal temperature; $T_{\text {sk: }}$ : Mean skin temperature; HR: Heart rate; MAP: Mean arterial blood pressure; $\mathrm{PaCO}_{2}$ : Arterial carbon dioxide tension; CSI: Cumulative stress index.}

\section{Acknowledgements}

The authors appreciate the time and effort expended by the volunteer subjects. We also thank Ms. Yoneya, Noma, Noguchi, Kanamaru, Imaizumi, and Yamashina for the recruitment of subjects and their support of this project.

\section{Authors' contributions}

MS, CGC, and SO conception and design of the research. MS, KS, AH, CGC, and SO performed experiments. MS analyzed the data. MS, KS, AH, TS, CGC, and SO interpreted the results of the experiments. MS prepared figures. MS, CGC, and SO drafted the manuscript and revisions thereof. All authors agreed to the final version submitted. All authors read and approved the final manuscript.

\section{Funding}

This study was supported in part by a Japan Society for the Promotion of Science KAKENHI Grant-in-Aid for Scientific Research 18H03166 (to M. Shibasaki), a Grant-in-Aid for Scientific Research $15 \mathrm{H03098}$ (to S. Ogoh) from the Japanese Ministry of Education, Culture, Sports, Science and Technology, and the National Institutes of Health GM068865 (to C.G. Crandall).

\section{Availability of data and materials}

All relevant data are within the paper.

\section{Ethics approval and consent to participate}

All procedures performed in studies involving human participants were in accordance with the ethical standards of the institutional and/or national research committee and with the 1964 Helsinki declaration and its later amendments or comparable ethical standards. All the participants signed the informed consent form agreeing to submit to the procedures involved in the study.

\section{Consent for publication}

Not applicable.

\section{Competing interests}

No conflict of interest, financial or otherwise, are declared by the authors.
Author details
${ }^{1}$ Department of Health Sciences, Faculty of Human Life and Environment, Nara Women's University, Kitauoya-Nishi Machi, Nara 630-8506, Japan.
2 Department of Health and Physical Education, Tokyo Gakugei University,
Tokyo, Japan. ${ }^{3}$ Department of Health and Welfare, Kyorin University, Tokyo, Japan. ${ }^{4}$ Research Institute of Physical Fitness, Japan Women's College of Physi- cal Education, Tokyo, Japan. ${ }^{5}$ Institute for Exercise and Environmental Medi- cine, Texas Health Presbyterian Hospital, Dallas, USA. ${ }^{6}$ Department of Internal Medicine, University of Texas, Southwestern Medical Center, Dallas, USA.
${ }^{7}$ Department of Biomedical Engineering, Toyo University, Saitama, Japan. 
Received: 13 November 2019 Accepted: 23 April 2020

Published online: 04 May 2020

\section{References}

1. Lind AR, Leithead CS, McNicol GW (1968) Cardiovascular changes during syncope induced by tilting men in the heat. J Appl Physiol 25:268-276

2. Schlader ZJ, Wilson TE, Crandall CG (2016) Mechanisms of orthostatic intolerance during heat stress. Auton Neurosci 196:37-46

3. Van Lieshout JJ, Wieling W, Karemaker JM, Secher NH (2003) Syncope, cerebral perfusion, and oxygenation. J Appl Physiol 94:833-848

4. Goswami N, Blaber AP, Hinghofer-Szalkay H, Convertino VA (2019) Lower body negative pressure: physiological effects, applications, and implementation. Physiol Rev 99:807-851

5. Bain AR, Morrison SA, Ainslie PN (2014) Cerebral oxygenation and hyperthermia. Front Physiol 5:92

6. Bain AR, Nybo L, Ainslie PN (2015) Cerebral vascular control and metabolism in heat stress. Compr Physiol 5:1345-1380

7. Kety SS, Schmidt CF (1946) Effects of alterations in the arterial tensions of carbon dioxide and oxygen on cerebral blood flow and cerebral oxygen consumption of normal young men. Fed Proc 5:55

8. Howden R, Lightfoot JT, Brown SJ, Swaine IL (2004) The effects of breathing $5 \% \mathrm{CO}_{2}$ on human cardiovascular responses and tolerance to orthostatic stress. Exp Physiol 89:465-471

9. Lucas RA, Pearson J, Schlader ZJ, Crandall CG (2013) Hypercapnia-induced increases in cerebral blood flow do not improve lower body negative pressure tolerance during hyperthermia. Am J Physiol Regul Integr Comp Physiol 305:R604-R609

10. Lewis NC, Bain AR, MacLeod DB, Wildfong KW, Smith KJ, Willie CK, Sanders ML, Numan T, Morrison SA, Foster GE, Stewart JM, Ainslie PN (2014) Impact of hypocapnia and cerebral perfusion on orthostatic tolerance. J Physiol 592:5203-5219

11. Ogoh S, Sato K, Okazaki K, Miyamoto T, Hirasawa A, Sadamoto T, Shibasaki M (2015) Blood flow in internal carotid and vertebral arteries during graded lower body negative pressure in humans. Exp Physiol 100:259-266

12. Sato K, Yoneya M, Otsuki A, Sadamoto T, Ogoh S (2015) Anatomical vertebral artery hypoplasia and insufficiency impairs dynamic blood flow regulation. Clin Physiol Funct Imaging 35:485-489

13. Ogoh S, Sato K, Okazaki K, Miyamoto T, Hirasawa A, Shibasaki M (2014) Hyperthermia modulates regional differences in cerebral blood flow to changes in CO2. J Appl Physiol 117:46-52

14. Ogoh S, Sato K, Okazaki K, Miyamoto T, Hirasawa A, Morimoto K, Shibasak M (2013) Blood flow distribution during heat stress: cerebral and systemic blood flow. J Cereb Blood Flow Metab 33:1915-1920

15. Shibasaki M, Namba M, Oshiro M, Kakigi R, Nakata H (2017) Suppression of cognitive function in hyperthermia; From the viewpoint of executive and inhibitive cognitive processing. Sci Rep 7:43528

16. Taylor WF, Johnson JM, Kosiba WA, Kwan CM (1989) Cutaneous vascular responses to isometric handgrip exercise. J Appl Physiol 66:1586-1592

17. Levine BD, Giller CA, Lane LD, Buckey JC, Blomqvist CG (1994) Cerebral versus systemic hemodynamics during graded orthostatic stress in humans. Circulation 90:298-306
18. Fan JL, Cotter JD, Lucas RA, Thomas K, Wilson L, Ainslie PN (1985) (2008) Human cardiorespiratory and cerebrovascular function during severe passive hyperthermia: effects of mild hypohydration. J Appl Physiol 105:433-445

19. Fujii N, Honda Y, Hayashi K, Kondo N, Koga S, Nishiyasu T (2008) Effects of chemoreflexes on hyperthermic hyperventilation and cerebral blood velocity in resting heated humans. Exp Physiol 93:994-1001

20. Wilson TE, Cui J, Zhang R, Crandall CG (2006) Heat stress reduces cerebral blood velocity and markedly impairs orthostatic tolerance in humans. Am J Physiol Regul Integr Comp Physiol 291:R1443-R1448

21. Schoning M, Walter J, Scheel P (1994) Estimation of cerebral blood flow through color duplex sonography of the carotid and vertebral arteries in healthy adults. Stroke 25:17-22

22. Tatu L, Moulin T, Bogousslavsky J, Duvernoy H (1996) Arterial territories of human brain: brainstem and cerebellum. Neurology 47:1125-1135

23. Sato K, Fisher JP, Seifert T, Overgaard M, Secher NH, Ogoh S (2012) Blood flow in internal carotid and vertebral arteries during orthostatic stress. Exp Physiol 97:1272-1280

24. Coverdale NS, Gati JS, Opalevych O, Perrotta A, Shoemaker JK (2014) Cerebral blood flow velocity underestimates cerebral blood flow during modest hypercapnia and hypocapnia. J Appl Physiol 117:1090-1096

25. Verbree J, Bronzwaer AS, Ghariq E, Versluis MJ, Daemen MJ, van Buchem MA, Dahan A, van Lieshout JJ, van Osch MJ (2014) Assessment of middle cerebral artery diameter during hypocapnia and hypercapnia in humans using ultra-high-field MRI. J Appl Physiol 117:1084-1089

26. van Helmond N, Johnson BD, Holbein WW, Petersen-Jones HG, Harvey RE, Ranadive SM, Barnes JN, Curry TB, Convertino VA, Joyner MJ (2018) Effect of acute hypoxemia on cerebral blood flow velocity control during lower body negative pressure. Physiol Rep 6:13594

27. Keller DM, Low DA, Wingo JE, Brothers RM, Hastings J, Davis SL, Crandall CG (2009) Acute volume expansion preserves orthostatic tolerance during whole-body heat stress in humans. J Physiol 587:1131-1139

28. Schlader ZJ, Seifert T, Wilson TE, Bundgaard-Nielsen M, Secher NH, Crandall CG (2013) Acute volume expansion attenuates hyperthermiainduced reductions in cerebral perfusion during simulated hemorrhage. J Appl Physiol 114:1730-1735

29. Lucas RAI, Wilson LC, Ainslie PN, Fan JL, Thomas KN, Cotter JD (2018) Independent and interactive effects of incremental heat strain, orthostatic stress, and mild hypohydration on cerebral perfusion. Am J Physiol Regul Integr Comp Physiol 314:R415-R426

30. Claassen JA, Meel-van den Abeelen AS, Simpson DM, Panerai RB, International Cerebral Autoregulation Research N (2016) Transfer function analysis of dynamic cerebral autoregulation: a white paper from the International Cerebral Autoregulation Research Network. J Cereb Blood Flow Metab 36:665-680

\section{Publisher's Note}

Springer Nature remains neutral with regard to jurisdictional claims in published maps and institutional affiliations.

Ready to submit your research? Choose BMC and benefit from

- fast, convenient online submission

- thorough peer review by experienced researchers in your field

- rapid publication on acceptance

- support for research data, including large and complex data types

- gold Open Access which fosters wider collaboration and increased citations

- maximum visibility for your research: over 100M website views per year

At BMC, research is always in progress.

Learn more biomedcentral.com/submissions 